\title{
OPTIMIZATION OF THE OPERATION RELIABILITY LEVEL OF THE PROTECTION SYSTEM
}

\author{
Marian CIONTU \\ Maria BROJBOIU \\ University of Craiova \\ Faculty of Electrical Engineering \\ str. Decebal, no.107, 1100, Craiova \\ phone: +40 251 435724, fax: +40 251 436447, e-mail: mciontu@elth.ucv.ro, mbrojboiu@elth.ucv.ro
}

\begin{abstract}
Ensuring the proper reliability level for the protection system is achieved by reserving the protection function.

An excessive enhance of the reservation level leads to decreasing of the system reliability, due to the more situations in which protection tempestuously operates.

The paper presents some considerations referring to definition of some indices regarding the protection system security.

There is approached the problem of determination of the multiplicity level for each of the component protections, so that the optimization of the ensemble security level to be ensured.

A more accurate analysis could be achieved by considering the corresponding phenomena as Markov processes.
\end{abstract}

\section{Key words}

Optimization; protection system; reliability

\section{Introduction}

The protection and control system is an important component of the power system and it has an essential contribution in assuring the continuity of consumers' supplying.

A characteristic of the electrical protection systems is the fact that the fault of an element does not have immediate consequences. This is detected only when the protected installation is in an operating regime that emphasizes the malfunction of the protection system. There are two types of protection faults: tempestuous operating and operating rejection.

The tempestuous operating of a protection means the protection system works under conditions it should not normally operate. This behavior is due to: modifying the adjustment values, increasing the gain of certain circuits, malfunctioning of input circuits. Under the specified circumstances, the protection works in an inadequate regime (e.g. a short-circuit protection working in an overload regime) or in case of a fault outside the protection area. The tempestuous operating of a protection leads to the supplying interruption of some consumers.

The operating rejection of a protection means that the system does not react in case of a fault in the protected area. The spare protection system located in the next junction towards the source will deal with this fault having as consequence the disconnection of certain circuits that normally shouldn't be affected by the fault. The energy supplying quality is altered in both cases.

Ensuring of the proper reliability level for the protection system is achieved by reserving the protection function. Creating a certain protection function is achieved using several equipments installed at the same junction.

It must be noted that the highly increasing of the reservation level leads to decreasing of the system reliability, due to the more situations in which protection tempestuously operates. By reserving the protection function decreasing the number of operating rejections, but also increases the number of tempestuous operations.

The paper presents some considerations referring to definition of some indices regarding the protection system security.

Hence, the problem is to determine the multiplicity level for each of the protection system component in order to ensure the optimization of the security level for the whole system. 


\section{Defining the reliability indices}

On this background, the following rates of events occurrence are defined in order to analyze the reliability of the system:

$\lambda_{n f}$ Rate of protection fault occurrence, leading to an operating rejection;

$\lambda_{f i}$ Rate of protection fault occurrence, that leading to tempestuous operating;

$\lambda_{r}$ rate of a certain fault occurrence in the protected system at which the protection should respond;

$\lambda_{i}$ rate of operating regime occurrence in the protected system at which the protection may inadvertently operate;

$\mu$ rate of protection restoration; after a malfunction occurrence all the defects of that mall operated protection will be removed.

The analysis of the operating regime between two successive faults is one of the system reliability approaches.

The following indicators can be computed.

The probability of protection fault occurrence in the $[0, t]$ interval, which leads to an operation denying:

$$
Q_{n f}(t)=1-e^{-\int_{0}^{t} \lambda_{n f} d t}=1-e^{-\lambda_{n f} t}
$$

The probability of protection fault occurrence in the $[0, t]$ interval, which leads to an inadvertent operation:

$$
Q_{f i}(t)=1-e^{-\int_{0}^{t} \lambda_{f i} d t}=1-e^{-\lambda_{f i} t}
$$

The operation rejection occurrence rate:

$$
\lambda_{r e f}=\lambda_{r} Q_{n f}(t)=\lambda_{r}\left[1-e^{-\lambda_{n f} t}\right]
$$

The tempestuous operation occurrence rate:

$$
\lambda_{\text {int }}=\lambda_{i} Q_{f i}(t)=\lambda_{i}\left[1-e^{-\lambda_{f i} t}\right]
$$

If there are $n$ independent protections, the previous rates have the following values:

$$
\begin{aligned}
& \lambda_{\text {ref }}=\lambda_{\text {ref }} \prod_{j=1}^{n}\left[1-e^{-\lambda_{n f j} t}\right] \\
& \lambda_{\text {int }}=\lambda_{i}\left[1-\prod_{j=1}^{n} e^{-\lambda_{f i j} t}\right]=\lambda_{i}\left[1-e^{-\left(\sum_{j=1}^{n} \lambda_{f i j}\right) t}\right]
\end{aligned}
$$

\section{Reliability analyses using Markov processes.}

A more accurate analysis could be achieved by corresponding phenomena according to Markov processes theory.

By analyzing the working regime of the system, the following assumptions can be made:

a) When a protection is affected by both a tempestuous operation fault and an operation rejection fault, as well, the latter is the one that activates the protection system.

b) The protected system has a fault detecting system that identifies the protections not properly operating after the fault has appeared.

c) When an inappropriate operation regime is detected, the faults of the protection installed in that junction are eliminated.

d) The period of time needed to restore the system is short, in order to avoid the occurrence of another fault during this time.

e) When an operation rejection or a tempestuous operating occurs, the circuit is not restored until at least a protection is repaired.

The transition graph (diagram) for a single protection is shown in figure 1 .

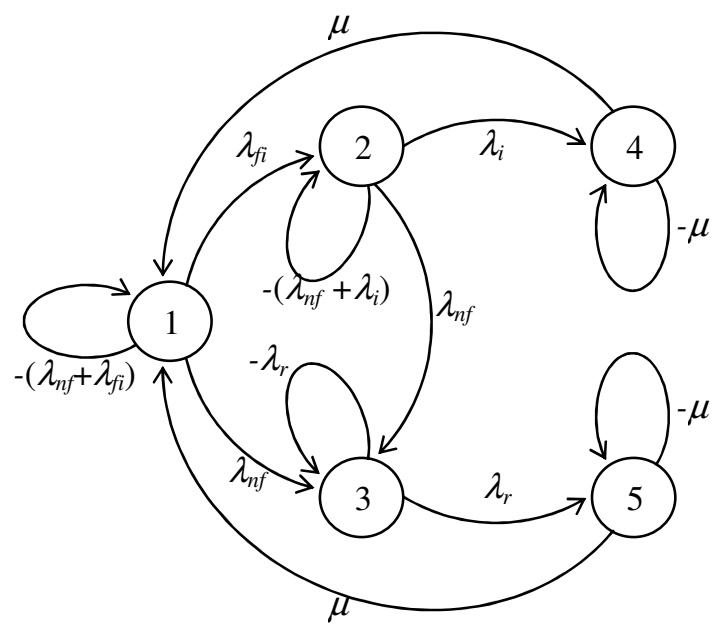

Fig.1. The transitions states graph for a single protection 
The states represented in this graph are:

1 - rated operation state, 2 - fault that produces tempestuous operation, 3 - fault that produces operation rejection, 4 - state of the tempestuous operation fault, 5 state of the operation rejection fault

By solving the following linear system, the probabilities $P_{1}$, $P_{2}, P_{3}, P_{4}, P_{5}$ of the graph states are obtained.

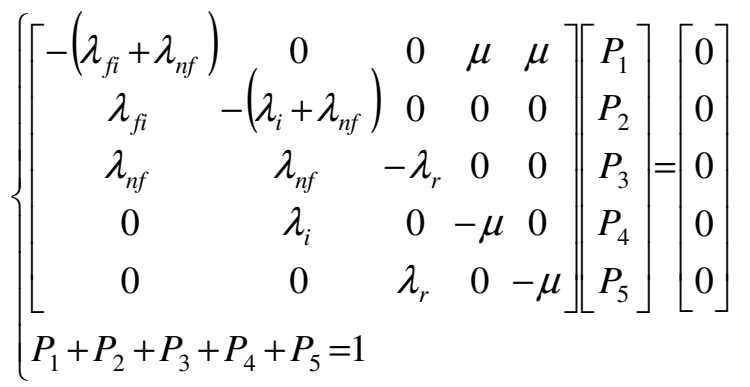

This computation method of the states probabilities is valid only if the reliability investigation time is great enough, so that the initial state of the system has no influence on the computed values.

For a single protection the achieved probabilities are as bellow.

The probability of tempestuous operation $P_{4}$ is:

$$
P_{i n t}=\frac{\lambda_{f i} \lambda_{i} \lambda_{r}}{\left(\lambda_{n f}+\lambda_{i}\right)\left[\left(\mu+\lambda_{f i}\right) \lambda_{r}+\left(\lambda_{r}+\mu\right) \lambda_{n f}\right]+\left(\lambda_{n f}+\lambda_{r}\right) \lambda_{f i} \mu}
$$

The probability of generation of operation rejection $P_{5}$ is:

$$
P_{r e f}=\frac{\lambda_{n f} \lambda_{r}\left(\lambda_{f i}+\lambda_{i}+\lambda_{n f}\right)}{\left(\lambda_{n f}+\lambda_{i}\right)\left[\left(\mu+\lambda_{f i}\right) \lambda_{r}+\left(\lambda_{r}+\mu\right) \lambda_{n f}\right)+\left(\lambda_{n f}+\lambda_{r}\right) \lambda_{f i} \mu}
$$

Probability of malfunction is achieved by adding the previous two values.

\section{Reliability analysis of a system with reservation level}

In the case of complex systems, comprising more protections, the analytical expressions of the computed probabilities are much elaborated. Due to this complexity, it is not possible to compare them in order to find the optimal value. The comparative analysis can be done by comparing the numerical values of the reliability indicators.

The transition graph of states for one system comprising two identical protections is shown in figure 2. As in the previous case, the computation of the state probabilities can be achieved after determining the transition rates matrix between distinct states.

Let us take as example a radial distribution system, as in figure 3 . We want to determine the optimal reservation level for the protection associated to the circuit breaker, labeled 1. The total power $\left(P_{t o t}\right)$ of the consumers, supplied from the above mentioned system is $P_{t o t}=19 \mathrm{MW}$. The power $P_{c 1}$ of the consumers connected to the analyzed circuit is $P_{c 1}=5 \mathrm{MW}$.

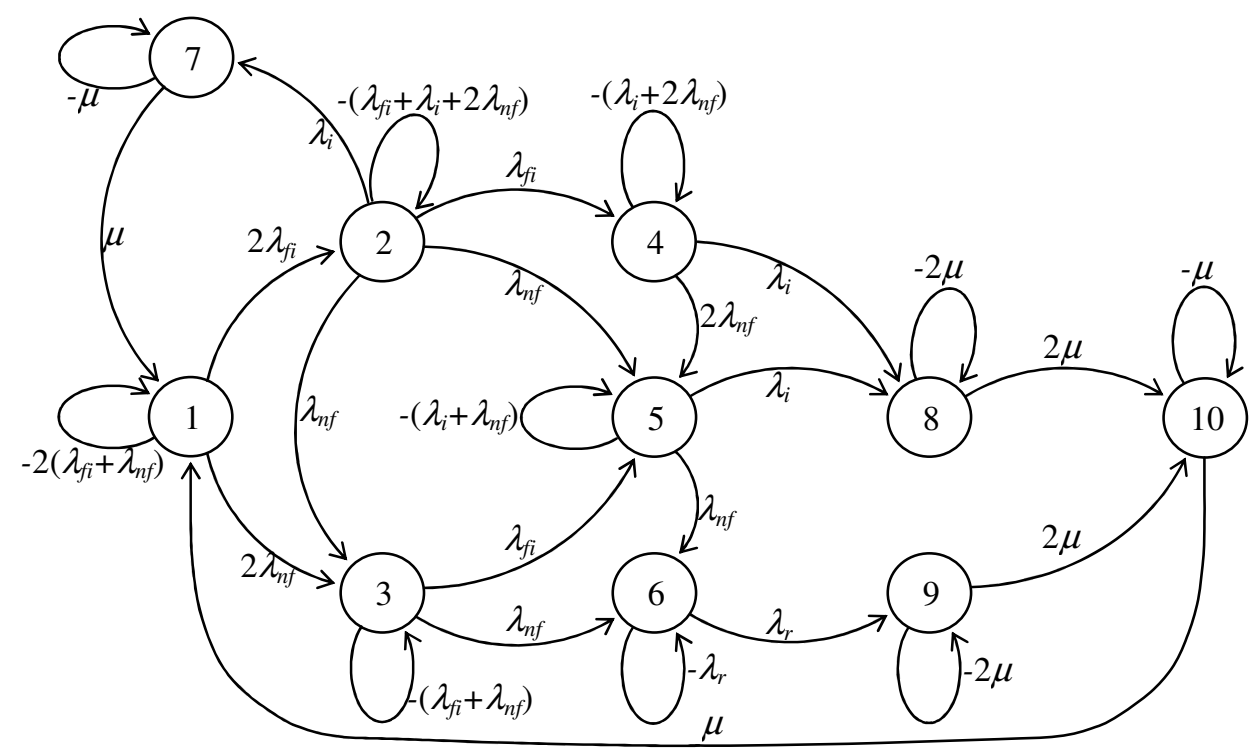

Fig.2. The transitions states graph for two protections 
TABLE 1

\begin{tabular}{|l|c|c|c|c|}
\hline \multirow{2}{*}{ Criteria } & \multirow{2}{*}{ Indicator } & \multicolumn{3}{|c|}{ Protection number } \\
\cline { 2 - 5 } & & 1 & 2 & 3 \\
\hline \multirow{2}{*}{$\begin{array}{l}\text { Malfunction operation } \\
\text { probability }\end{array}$} & $P_{\text {int }}$ & $2.492 \cdot 10^{-5}$ & $1,427 \cdot 10^{-5}$ & $4.07 \cdot 10^{-5}$ \\
\cline { 2 - 5 } & $P_{\text {ref }}$ & $4.991 \cdot 10^{-5}$ & $2,847 \cdot 10^{-5}$ & $0.666 \cdot 10^{-5}$ \\
\cline { 2 - 5 } & $P_{\text {tot }}$ & $7.483 \cdot 10^{-5}$ & $4.274 \cdot 10^{-5}$ & $4.736 \cdot 10^{-5}$ \\
\hline \multirow{2}{*}{$\begin{array}{l}\text { The average probable number } \\
\text { of malfunction operation in }\end{array}$} & $N_{\text {int }}$ & 0,437 & 0,624 & 0,83 \\
\cline { 2 - 5 } 10 years & $N_{\text {ref }}$ & 0,875 & 0,5 & 0,35 \\
\cline { 2 - 5 } & $N_{\text {tot }}$ & 1,312 & 1,124 & 51,18 \\
\hline Undelivered energy & $E_{n}[\mathrm{MWh}]$ & 144,25 & 59,94 & 58 \\
\hline
\end{tabular}

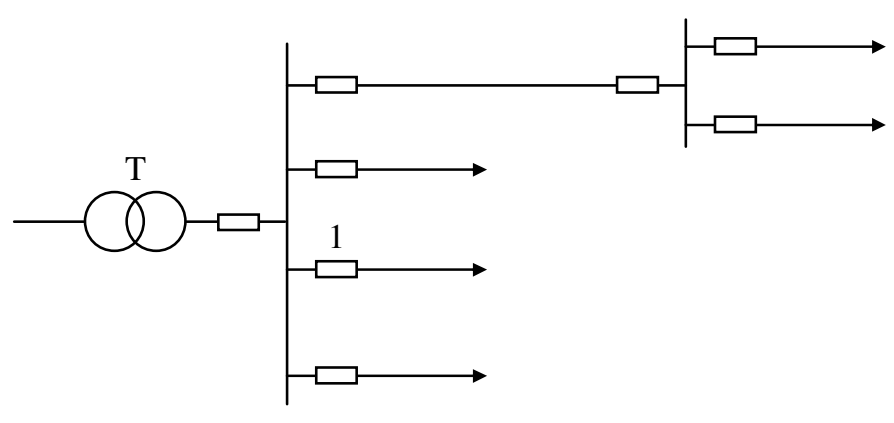

Fig.3. Supplying system example

The following values of the event generation rates are known:

$$
\begin{array}{ll}
\lambda_{f i}=0.05 \cdot 10^{-4}\left[\mathrm{~h}^{-1}\right] ; & \lambda_{n f}=0.1 \cdot 10^{-4}\left[\mathrm{~h}^{-1}\right] \\
\lambda_{i}=100 \cdot 10^{-4}\left[\mathrm{~h}^{-1}\right] ; & \lambda_{r}=60 \cdot 10^{-4}\left[\mathrm{~h}^{-1}\right] \\
\mu=2000 \cdot 10^{-4}\left[\mathrm{~h}^{-1}\right] ; &
\end{array}
$$

In order to determine the optimal solution, we can consider several objective functions, which must be either maximized or minimized.

a. Minimizing the malfunction operation probability

The probabilities of a tempestuous operation and rejection operation are obtained by adding the probabilities of the states corresponding to this situation. In figure 3 , the states denoted 7 and 8 correspond to a tempestuous operation and state 9 corresponds to a rejection operation.

\section{b. Minimizing the number of malfunction operations}

The probable average number of malfunction operation in the protection system, corresponding to the reference period $T$, can be determined. For the system comprising two protections we can compute:

- the average probable number of tempestuous operations

$$
N_{\text {int }}=\left(P_{2}+P_{4}+P_{5}\right) \lambda_{i} T
$$

- the average probable number of operation rejections

$$
N_{\text {int }}=P_{4} \lambda_{r} T
$$

c. Minimizing the power not supplied to the consumers
The probable amount of power that is not supplied to the consumers in the reference period $T$, due to the malfunction operation of the protection system, can be computed using the formula:

$$
E_{n}=T\left[P_{c 1} P_{\mathrm{int}}+\left(P_{t o t}-P_{c 1}\right) P_{r e f}\right]
$$

d. The global economical analysis taking into account the undelivered power, the losses produced to the consumers and the cost of the additional protection equipment.

The results obtained are presented in table 1 . A system with two protections is recommended when comparing the solutions from the malfunction operation probability or average number of operation point of view. The use of an additional protection is not in our benefit. From the undelivered power point of view, it is advantageous to use three protections in the protected circuit. Using the economical analysis criteria, we draw the conclusion that a single protection is appropriate. The economical advantages of an approximately $84 \mathrm{MWh}$ additional supply to the consumers do not compensate for the cost of installing an additional protection.

\section{Conclusions}

Obtaining an optimal structure for the protection system assumes determining the optimal level of reservation for each piece of equipment. Solving this problem needs complex analysis from both technical and economical point of view. Technical aspects can prevail in certain circumstances (e.g. important consumers, which cannot tolerate supplying interruptions), while in other circumstances the economical aspects are more important. Hence, a general solution cannot be presented; each case should be separately analyzed.

\section{References}

[1] Momoh, J. Electric Power System Applications of Optimisation, Marcel Dekker, New York, 2001

[2] Ionescu, D.C., Limnios, N. Statistical and Probabilistic Models in Reliability, Birkhauser, Boston, 1999

[3] Gnedenko, B., Ushakov, I., Falk, J. Probabilistic Reliability Engineering, John Wiley \&Sons, England, 1995 